\title{
Method for Estimating Activity Coefficients of Target Components in Poorly Specified Mixtures
}

Fabian Jirasek ${ }^{\dagger}$,Jakob Burger ${ }^{\ddagger}$, Hans Hasse*, ${ }^{*}$

${ }^{\dagger}$ Laboratory of Engineering Thermodynamics (LTD), University of Kaiserslautern, Erwin-

5 schrödinger-Straße 44, 67663 Kaiserslautern, Germany.

${ }^{+}$Chair for Chemical Process Engineering, Technical University Munich, Schulgasse 16, 94315

Straubing, Germany.

*hans.hasse@mv.uni-kl.de 
15 Abstract: Mixtures that contain a known target component but are otherwise poorly specified are important in many fields. Previously, the activity of the target component, which is needed e.g. to design separation processes, could not be predicted in such mixtures. A method was developed to solve this problem. It combines a thermodynamic group contribution method for the activity coefficient with NMR spectroscopy, which is used for estimating the nature and

20 amount of the different chemical groups in the mixture. The knowledge of the component speciation of the mixture is not required. Test cases that are inspired by bioprocess engineering applications show that the new method gives surprisingly good results.

Introduction: Liquid mixtures that contain a known target component but are otherwise poorly

25 specified are common in bioprocess engineering but also in many other fields of engineering and science. The thermodynamic activity of the target component is a key property that is needed, e.g., to calculate partition coefficients for designing separation processes. There are two established approaches for predicting activity coefficients: the phenomenological group contribution method UNIFAC ${ }^{1-4}$ and COSMO-RS ${ }^{5,6}$, which is based on a quantum chemical

30 description of the components of the mixture. In their present versions, both require information on the full speciation of the mixture, so that they cannot be applied directly to the prediction of activity coefficients in poorly specified mixtures.

NMR spectroscopy can be used for determining the speciation of complex mixtures. However, this is not always possible. In particular, overlapping signals and uncertainties in the assignment 35 of signals to the species in the mixture may cause problems. The position of an NMR signal in the spectrum depends on the chemical group in which the nucleus is located. Hence, there are 
regions in the NMR spectrum that can be assigned to different chemical groups. From an evaluation of the signals in such regions, quantitative information on the chemical groups in a mixture can be obtained. This is much simpler than determining the concentration of individual

40 components based on a peak assignment. The information on the types and quantities of groups in a mixture determined from the NMR spectrum is a suitable input for thermodynamic group contribution methods. It is demonstrated here that it can be used for determining activity coefficients of target components in the mixture using the UNIFAC method. The new method is called NEAT: NMR spectroscopic method for estimating activity coefficients of known target

45 components in poorly specified mixtures.

In NEAT, it is assumed that the components of the mixture are divided in two classes: one for which the components' nature and concentration are known, and one for which this is not the case. The target component belongs to the first class and may be its only member. The information on the components of the first class may stem from any source, in particular also 50 from NMR spectroscopy.

Estimating thermodynamic properties in poorly specified mixtures is not an entirely new field. Previous authors focused mostly on petrochemical systems ${ }^{7-14}$ and wood $\operatorname{tars}^{15}$ and applied a pseudo-component approach. This differs substantially from the NEAT approach, which aims at predicting a property of a single target component using a group contribution method.

55 Furthermore, the field for which NEAT was developed is bioprocess engineering, and various aqueous systems are studied here as examples. However, the NEAT method is not restricted to that field. 
Method: The key task in NEAT is to map the information on groups obtained from NMR

60 spectroscopy to the groups used in the thermodynamic group contribution method. There are different ways to solve this task. The choice will depend on the type of mixture that is studied, the type of NMR spectroscopy that is applied, and the groups for which parameters are available in the group contribution method. Hence, NEAT describes an idea, not a fixed algorithm. This idea is illustrated here using several examples. All studied systems are aqueous solutions of 65 organic components, in which one component is designated as target $(\mathrm{T})$. The concentration (mass fraction or molality) of that component and that of water (W) is assumed to be known, no information on the other components, which are summarily designated as unknown (U), is used. The examples that are presented here are only a small but representative part of a large study, which will be published elsewhere.

70 One-dimensional ${ }^{13} \mathrm{C}$ NMR spectroscopy was used in the present work for obtaining the information on the nature and concentration of chemical groups in the mixture. The ${ }^{13} \mathrm{C}$ NMR spectrum of the studied mixture was divided into different regions of chemical shift to which chemical groups were assigned. The choice of the chemical groups and their assignment to the different regions in the spectrum is shown in Table 1. The chemical groups correspond directly

75 to subgroups from the UNIFAC parameter table, which are referred to simply as groups here. The UNIFAC version used is UNIFAC (Dortmund) ${ }^{3,4}$. The assignment shown in Table 1 was used for all test systems. The concentrations of the groups for which NMR signals are obtained (cf. Table 1) are calculated from the NMR data. It is shown in the Supporting Information that mass balances can be used to obtain also the concentration of the lumped groups for which no 80 signals are obtained, which are assumed to be hydroxyl groups (cf. Table 1). 
Table 1. Assignment of ${ }^{13} \mathrm{C}$ NMR chemical shift regions to chemical groups used in the present work in NEAT. In the last column, examples for other chemical groups with signals in the same chemical shift region are given. In NEAT, these groups are, if present, represented by the 85 selected UNIFAC group.

\begin{tabular}{|l|l|l|l|}
\hline $\begin{array}{l}{ }^{13} \mathrm{C} \text { NMR } \\
\text { chemical shift } \\
\text { region }\end{array}$ & $\begin{array}{l}\text { Selected chemical } \\
\text { group name }\end{array}$ & UNIFAC label & $\begin{array}{l}\text { Examples for other chemical } \\
\text { groups with signals in the same } \\
\text { region }\end{array}$ \\
\hline $0-60$ & Alkyl group & $\mathrm{CH} 2$ & Cycloalkyl group, ether group \\
\hline $60-90$ & Alcohol group & $\mathrm{CH}+\mathrm{OH}(\mathrm{P})$ & Ether group, alkynyl group \\
\hline $90-150$ & Alkenyl group & $\mathrm{CH}=\mathrm{CH}$ & Aromatic carbon, cyano group \\
\hline $150-180$ & Carboxyl group & $\mathrm{COOH}$ & Ester group, amide group \\
\hline$>180$ & Carbonyl group & $\mathrm{CH} 2 \mathrm{CO}$ & (Only exceptional cases) \\
\hline n.a. & Hydroxyl group & $\mathrm{OH}(\mathrm{P})$ & Any group w/o carbon \\
\hline
\end{tabular}

The version of UNIFAC that is used here distinguishes between more than 100 groups, whereas in our examples in NEAT only six groups are used, which have to be groups from the UNIFAC list. The corresponding assignment does not have to be fully correct in order to obtain

90 good results. E.g., if an NMR signal is assigned to some polar group P, but is caused partly or entirely by some other chemically similar polar group $\mathrm{P}^{*}$, the resulting error in the prediction of the activity coefficient of the target component may not be large.

In UNIFAC, activity coefficients are modeled as a sum of two contributions, usually referred to as residual and combinatorial parts. The residual part plays the key role and contains group 95 interaction parameters, which are used in connection with group surface area fractions. The group surface area fractions are determined from group mole fractions and numbers for the group surface areas, which are taken from the UNIFAC parameter table. In NEAT, the group mole 
fractions are determined by NMR spectroscopy, such that the residual part is known. The NMR spectroscopic information on the groups is also used for calculating the combinatorial part, but

100 for that calculation, additionally, an estimate for the mean molar mass $M_{\mathrm{U}}$ of the unspecified components is needed. That estimate is also needed to calculate the mole fractions $x_{\mathrm{T}}$ and $x_{\mathrm{W}}$ of the target component and water from the respective mass fractions or molalities. For the examples discussed here, the choice of $M_{\mathrm{U}}$ has little influence on the results for the activity coefficient of the target component as long as no unreasonably small values are chosen. In the 105 present study, $M_{\mathrm{U}}=150 \mathrm{~g} / \mathrm{mol}$ is used throughout for the calculations with NEAT. For more information including a numerical example for the application of NEAT, see Supporting Information.

Results and Discussion: In the following, results for test systems consisting of a target 110 component (T), water (W), and additional components (U) are discussed. The studied mixtures were fully specified by gravimetric preparation, but for the application of the NEAT method, only the knowledge on the target component (T) and water (W) was used, but not that on the remaining components (U). The activity coefficient of the target component $\gamma_{\mathrm{T}}$ as predicted by NEAT for the poorly specified mixture is compared to that obtained from UNIFAC for the fully 115 specified mixture. For simplicity, only data for $298 \mathrm{~K}$ and 1 bar is considered.

The plots in which the results are presented are the same for all studied systems. The activity coefficient of the target component $\gamma_{\mathrm{T}}$ is shown as a function of the mole fraction $x_{\mathrm{U}}=1-x_{\mathrm{T}}-$ $x_{\mathrm{W}}$ of the components $\mathrm{U}$, for which no information was used in NEAT, for a constant molar ratio of $\mathrm{T} / \mathrm{W}$. For $x_{\mathrm{U}}=0$, the result from NEAT (not plotted) and that from UNIFAC must be the 120 same. The test for NEAT is whether it can predict the influence of $U$ on $\gamma_{\mathrm{T}}$. 
Figure 1 shows results for two ternary mixtures containing a target component $(\mathrm{T}=$ citric acid or ethanol), water (W), and D-glucose (U). The influence of $U$ on $\gamma_{\mathrm{T}}$ is strong for $\mathrm{T}=$ citric acid but weak for $\mathrm{T}=$ ethanol. Both trends are predicted well by the NEAT method.

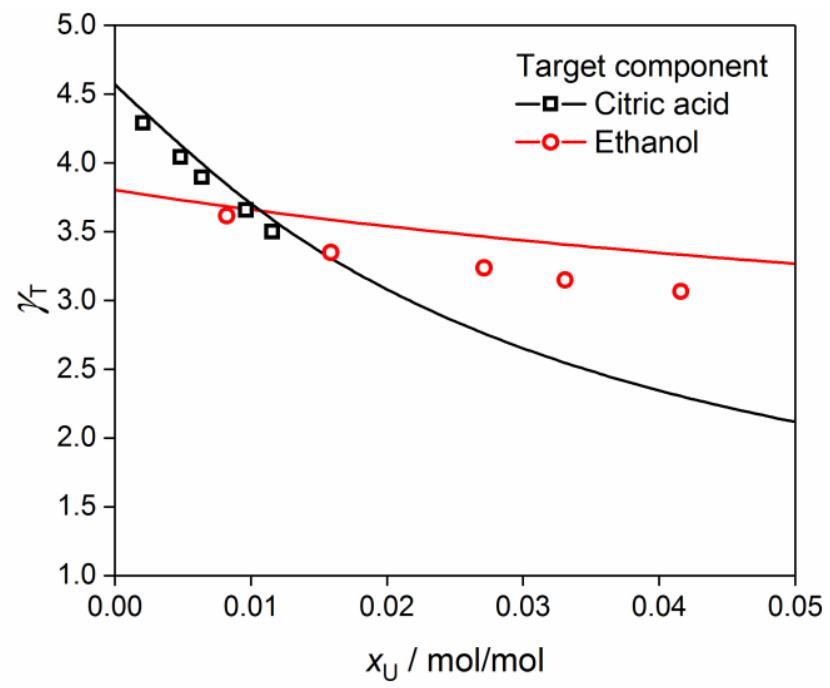

125 Figure 1. Activity coefficients $\gamma_{\mathrm{T}}$ of target components $(\mathrm{T}=$ citric acid or ethanol $)$ in ternary mixtures of T with water (W) and D-glucose (U) at $298 \mathrm{~K}$ and 1 bar. The molar ratio of T / W is 0.011 for $\mathrm{T}=$ citric acid and 0.049 for $\mathrm{T}=$ ethanol. No information on D-glucose $(\mathrm{U})$ was used in the NEAT method. Lines: results from UNIFAC for the fully specified mixture. Symbols: predictions with the NEAT method.

130 In Figure 2, results for two five-component systems are shown: (1,4-butanediol - water cyclohexanone - acetonitrile - methyl acetate) and (acetone - water - D-xylose - acetic acid methyl acetate). In the first system, the target component (T) is 1,4-butanediol, in the second it is acetone. In both systems, water (W) is the known solvent. The remaining three components are considered as unknown (U) in NEAT. The total mole fraction of all unspecified components

135 varies, while the mass ratio of the three unspecified components is always 1:1:1. Figure 2 shows that the influence of $U$ on the activity coefficient of the target components $\gamma_{\mathrm{T}}$ is predicted very well by the NEAT method in both cases. In the Supporting Information, results from a sensitivity 
analysis are presented, in which the influence of the uncertainty of the mass fraction of the target component on the results for the activity coefficient of the target component was studied. It shows that the influence is not much larger than that observed for a corresponding study with UNIFAC alone. In the example, a variation of the mass fraction of the target component of $10 \%$ leads to variations of the activity coefficient of about $3 \%$.

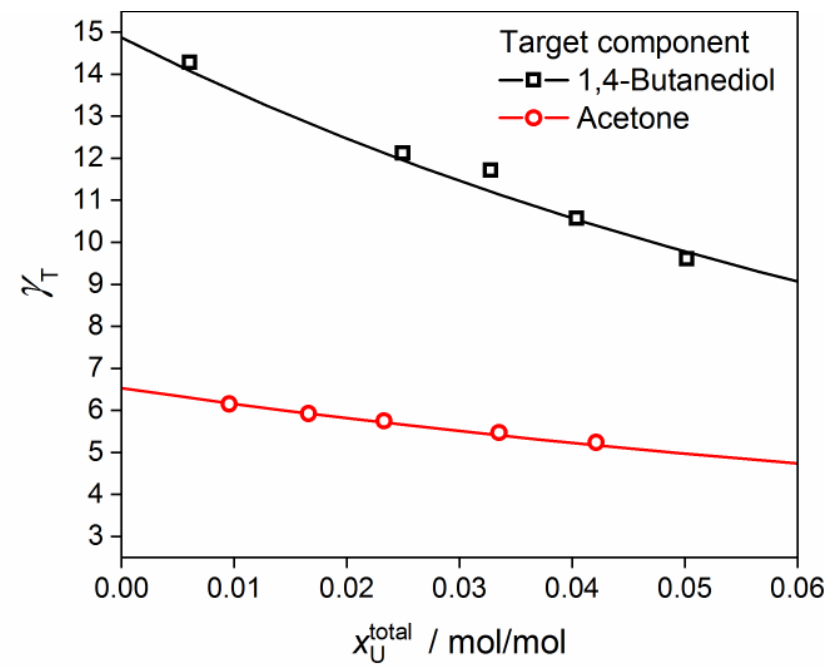

145 Figure 2. Activity coefficients $\gamma_{\mathrm{T}}$ of target components $(\mathrm{T}=1,4$-butanediol or acetone) in fivecomponent mixtures of $\mathrm{T}$ with water $(\mathrm{W})$ and a mixture of three unspecified components $(\mathrm{U}=$ cyclohexanone, acetonitrile, methyl acetate) or ( $\mathrm{U}=\mathrm{D}$-xylose, acetic acid, methyl acetate) at $298 \mathrm{~K}$ and 1 bar. The mass ratio of the three unspecified components is always 1:1:1. The molar ratio of $\mathrm{T} / \mathrm{W}$ is 0.022 for $\mathrm{T}=1,4$-butanediol and 0.040 for $\mathrm{T}=$ acetone. No information on the unspecified components (U) was used in the NEAT method. Lines: results from UNIFAC for the fully specified mixture. Symbols: predictions with the NEAT method.

Figure 3 shows results for three ternary systems: (ethanol - water - acetic acid), (ethanol water - methyl acetate), and (ethanol - water - 2-butanone). The target component (T) is always ethanol but the nature of the unspecified component (U), for which no information is used in 
155 NEAT, varies: $(\mathrm{U}=$ acetic acid, methyl acetate, or 2-butanone, respectively). As the molar ratio $\mathrm{T} / \mathrm{W}$ is the same for all systems, also the number for $\gamma_{\mathrm{T}}$ obtained for $x_{\mathrm{U}}=0$ is the same. The influence of $\mathrm{U}$ on $\gamma_{\mathrm{T}}$ differs strongly for the different studied components $\mathrm{U}$. Acetic acid shows the smallest, methyl acetate a medium, and 2-butanone the largest influence. In all cases, the predictions with the NEAT method match the UNIFAC results very well. This shows clearly that 160 the NEAT method is able to distinguish unknowns of different chemical nature.

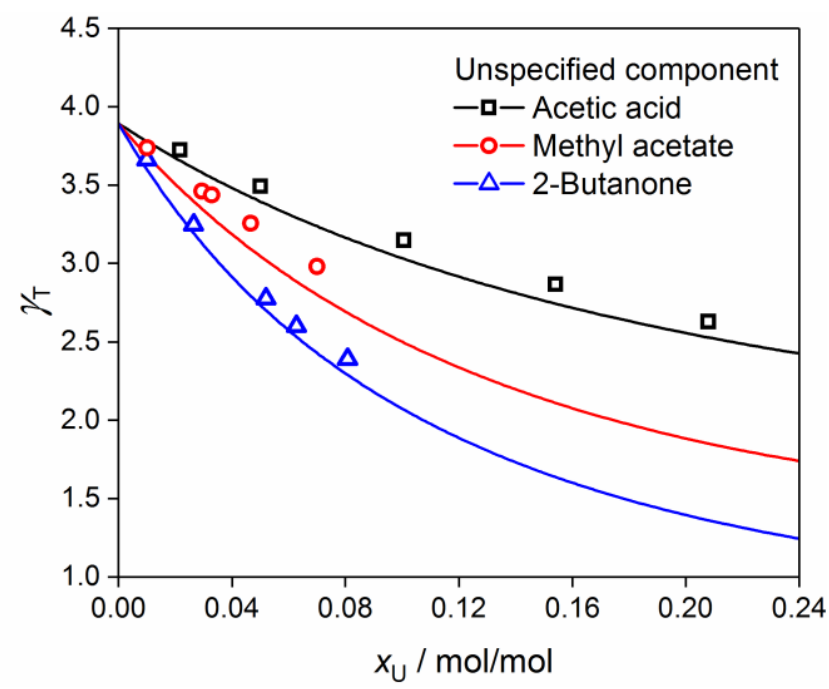

Figure 3. Activity coefficient $\gamma_{\mathrm{T}}$ of target component $(\mathrm{T}=$ ethanol $)$ in ternary mixtures of $\mathrm{T}$ with water (W) and an unspecified component ( $\mathrm{U}$ = acetic acid, methyl acetate, or 2-butanone) at $298 \mathrm{~K}$ and 1 bar. The molar ratio of $\mathrm{T} / \mathrm{W}$ is 0.044 for all systems. No information on the unspecified components $(\mathrm{U})$ was used in the NEAT method. Lines: results from UNIFAC for the fully specified mixture. Symbols: predictions with the NEAT method.

Only a few examples for applications of the NEAT method were presented here. They are, however, representative for results that were obtained in extensive studies of mixtures of different types. In those studies, results from NEAT were not only compared to those from UNIFAC, as it was done here, but also to experimental data and good agreement was found. The 
NEAT method is not restricted to ${ }^{13} \mathrm{C}$ NMR spectroscopy. Also other NMR techniques can be used, and different NMR techniques can be combined. Moreover, also results from other spectroscopic methods and other methods for calculating the activity coefficient such as COSMO-RS ${ }^{5}$ or F-SAC ${ }^{16}$ can be applied. A strength of the NEAT method is that it can be

175 adapted to specific situations. Basically any available quantitative information on the speciation of the mixture can be incorporated. Also qualitative information helps, e.g. for establishing the assignment of the NMR signals to the groups. The idea behind NEAT is the combination of the spectroscopic determination of a group speciation with thermodynamic methods. It can in principle be applied to any field of thermodynamics for which group contribution methods are 180 available.

\section{Associated Content}

Supporting Information: Materials, methods, example for the application of NEAT, results for the influence of the molar mass of the unspecified components, results for the influence of the 185 uncertainty of the concentration of the target component.

\section{Author Information}

Corresponding Author: *hans.hasse@mv.uni-kl.de

Notes: The authors declare no competing financial interests. 


\section{Acknowledgments}

The authors gratefully acknowledge financial support from the European Union's Framework 195 Program for Research and Innovation under grant agreement no. 637077 (PRODIAS).

\section{References}

(1) Fredenslund, A.; Jones, R. L.; Prausnitz, J. M. Group-Contribution Estimation of Activity Coefficients in Nonideal Liquid mixtures. AIChE J. 1975, 21(6), 1086-1099.

200 (2) Fredenslund, A.; Gmehling, J; Rasmussen, P. Vapor-Liquid Equilibria using UNIFAC, a Group-Contribution Method; Elsevier: Amsterdam, Netherlands, 1977.

(3) Weidlich, U.; Gmehling, J. A Modified UNIFAC Model, Part I: Prediction of VLE, $\mathrm{h}_{\mathrm{E}}$ and $\gamma_{\infty}$. Ind. Eng. Chem. Res. 1987, 26, 1372-1381.

(4) Constantinescu, D.; Gmehling, J. Further Development of Modified UNIFAC

205 (Dortmund): Revision and Extension. J. Chem. Eng. Data 2016, 61(8), 2738-2748.

(5) Klamt A. Conductor-Like Screening Model for Real Solvents: A New Approach to the Quantitative Calculation of Solvation Phenomena. J. Phys. Chem. 1995, 99, 2224-2235.

(6) Klamt, A.; Jonas, V.; Bürger, T.; Lohrenz, J. C. Refinement and Parametrization of COSMO-RS. J. Phys. Chem. A 1998, 102, 5074-5085.

210 (7) Ruzicka Jr, V.; Fredenslund, A.; Rasmussen, P. Representation of Petroleoum Fractions by Group Contribution. Ind. Eng. Chem. Process Des. Dev. 1983, 22, 49-53.

(8) Alexander, G. L.; Creagh, A. L.; Prausnitz, J. M. Phase Equilibria for High-Boiling Fossil-Fuel Distillates. 1. Characterization. Ind. Eng. Chem. Fundam. 1985, 24, 301-310. 
(9) Alexander, G. L.; Schwarz, B. J.; Prausnitz, J. M. Phase Equilibria for High-Boiling Fossil-Fuel Distillates. 2. Correlation of Equation-of-State Constants with Characterization Data for Phase-Equilibrium Calculations. Ind. Eng. Chem. Fundam. 1985, 24, 311-315.

(10) Hartounian, H.; Allen, D. T. Group Contribution Methods for Coal-Derived Liquids: Hydrogen Solubilities using a UNIFAC Approach. Fuel 1988, 67(12), 16091614.

(11) Carreón-Calderón, B.; Uribe-Vargas, V.; Ramírez-Jaramillo, E.; Ramírez-deSantiago, M. Thermodynamic Characterization of Undefined Petroleum Fractions Using Group Contribution Methods. Ind. Eng. Chem. Res. 2012, 51, 14188-14198.

Fahim, M. A.; Elkilani, A. S. Prediction of Solubility of Hydrogen in Petroleum Cuts using Modified UNIFAC. Can. J. Chem. Eng. 1992, 70(2), 335-340. Darwish, E.; Al-Sahhaf, T. A.; Fahim, M. A. Prediction of the Surface Tension of Petroleum Cuts using a Modified UNIFAC Group Contribution Method. Fluid Phase Equilib. 1995, 105(2), 229-239. Jameel, A. G. A.; Elbaz, A. M.; Emwas, A.-H.; Roberts, W. L.; Sarathy, S. M. Calculation of Average Molecular Parameters, Functional Groups, and a Surrogate Molecule for Heavy Fuel Oils using 1H and 13C Nuclear Magnetic Resonance Spectroscopy. Energy Fuels 2016, 30(5), 3894-3905.

Thermodynamic Property Estimation for Wood Tars: A Functional Group Approach. Liq. Fuels Technol. 1984, 2(3), 327-353. De P. Soares, R.; Gerber, R. P. Functional-Segment Activity coefficient Model. 1. Formulation. Ind. Eng. Chem. Res. 2013, 52(32), 11159-11171. 
TOC Graphic

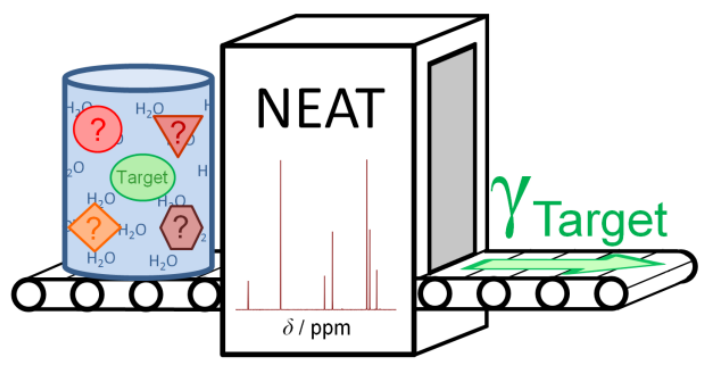

